Ann. Biol. anim. Bioch. Biophys., I971, 11 (I), 55-68.

\title{
LES RÉCEPTEURS RESPONSABLES DE L'HYPERMAGNÉSÉMIE CONSÉCUTIVE A UNE INJECTION DE CATÉCHOLAMINES
}

\author{
P. LARVOR et Y. RAYSSIGUIER \\ Station de Physiopathologie de la Nutrition, \\ Centre de Recherches zootechniques et vétérinaives, I. N.R. A., \\ 63 - Saint-Genès-Champanelle
}

\section{RÉSUMÉ}

L'hypermagnésémie obtenue chez le Rat par injection de substances adrénergiques (adrénaline ou isoprénaline) semble être la conséquence d'une stimulation de $\beta$ récepteurs. L'action de l'adrénaline est nettement plus puissante que celle de l'isoprénaline et le blocage complet de l'hypermagnésémie adrénalinique n'est possible que par l'association de phentolamine et de propranolol d'où la notion de $\beta$ récepteurs atypiques dont l'existence a d'ailleurs été envisagée chez le Rat pour d'autres effets métaboliques des catécholamines. L'hypermagnésémie adrénalinique qui peut être dissociée de la glycogénolyse hépatique et au moins en partie de la glycogénolyse musculaire n'a pas pour origine ni pour relais les viscères abdominaux.

\section{INTRODUCTION}

L'injection intrapéritonéale d'une forte dose d'adrénaline provoque une hypermagnésémie importante chez le Rat (LARvor, I968). Bien que la signification physiologique de cette réaction soit incertaine, son étude plus approfondie devrait permettre d'explorer le mode de régulation de la magnésémie très mal connu actuellement. Nous nous sommes attachés à préciser la nature des récepteurs adrénergiques responsables de l'élévation de la magnésémie, à la fois sous l'aspect de leur type physiologique, de leur liaison avec les autres phénomènes métaboliques et de leur localisation anatomique.

Classiquement les récepteurs cellulaires qui réagissent à la stimulation par les catécholamines sont classés en deux grandes catégories : les récepteurs $\alpha$, responsables de la contraction de certaines fibres musculaires lisses et de l'augmentation de la tension artérielle, et les récepteurs $\beta$ qui sont à l'origine des effets métaboliques (glycogénolyse hépatique et musculaire), de l'effet inotrope cardiaque et de la relaxa- 
tion de certaines fibres musculaires lisses. Les effets $\beta$ ont pour médiateur la sti mulation de l'adényl-cyclase et la formation d'adénosine monophosphate cyclique dans certaines cellules ou parties de cellules (Robison, BuTcher et SutherLand, I967). $L$ 'absence d'action de la noradrénaline sur la magnésémie du Rat semble indiquer plutôt des récepteurs $\beta$ adrénergiques mais ceci est en contradiction avec le blocage par la dihydroergotamine (LARVOR, I968) qui inhibe surtout les effets $\alpha$. Cependant il est démontré (ELLIS, I967) que le Rat se comporte de façon assez particulière en ce qui concerne les effets métaboliques des catécholamines. Ainsi 1'hyperglycémie adrénalinique, effet $\beta$ typique chez la plupart des espèces est peu sensible chez le Rat. aux bloqueuts $\beta$ spécifiques mais est inhibée par la dihydroergotamine. Les récepteurs adrénergiques peuvent être caractérisés par le pouvoir relatif de l'adrénaline (activateurs $\alpha$ et $\beta$ ), de la noradrénaline (activateur principalement $\alpha$ ) et de l'isoprénaline (activateur essentiellement $\beta$ ) et par la possibilité de divers bloqueurs de s'opposer à l'effet envisagé : la dihyđ̛oergotamine étant un bloqueur principalement $\alpha$, la phentolamine (Régitine $(\mathbf{R})$ ) un bloqueur $\alpha$ pur et le propranolol (Avlocardyl $(\mathbf{R})$ ). un bloqueur $\beta$ pur.

\section{MATÉRIEL ET MÉTHODES}

Des rats de souche Sherman, non à jeun, des deux sexes et du même élevage sont répartis au hasard dans les groupes nécessaires aux essais. Pour une même expérience ils sont choisis de même sexe et de poids voisin.

\section{I. - Étude pharmacolque}

Pour l'étude pharmacologique, toutes les injections sont intrapéritonéales et les témoins reçoivent un volume de sérum physiologique équivalent à la quantité injectée aux animaux traités. Le bloqueur est injecté 30 minutes avant l'injection de catécholamines. Le sang est prélevé par ponction cardiaque sous anesthésie à l'éther, $60 \mathrm{mn}$ après l'administration de L-adrénaline ou d'isoprénaline (= isoprotérénol) (Aleudrine $(\mathbf{R})$ ).

\section{2. - Localisation anatomique des récepteurs}

Durant ces essais, l'éviscération abdominale, la surrénalectomie et la néphrectomie ont été réalisées selon la technique générale décrite par INGLE et GrifFith (Ig63) et l'hépatectomie partielle selon KABAL et RAMEY (1965).

- Éviscération abdominale: elle n'est que partielle, le foie, les reins et les surrénales restant en place. En fin d'intervention, les animaux après une prise de sang par ponction cardiaque reçoivent une injection sous-cutanée de $2 \mathrm{mg} / \mathrm{kg}$ d'adrénaline et les témoins un volume de séru $\mathrm{m}$ physiologique équivalent. Une nouvelle prise de sang est effectuée une heure après l'injection.

- Hépatectomie: les rats sont utilisés 24 heures après une hépatectomie partielle. Des animaux " pseudo-opérés ", servent de témoins. Le pourcentage de foie enlevé est calculé de la façon suivante : la portion enlevée est pesée et le lendemain, après l'expérience, les animaux sont abattus et le reste du foie est pesé, ce qui permet de calculer un pourcentage d'ablation :

$$
\frac{\text { portion enlevée }}{\text { poids total }} \times \text { roo. }
$$

Le sang est prélevé avant et une heure après l'injection đe $2 \mathrm{mg} / \mathrm{kg}$ d'adrénaline par voie sous-cutanée.

- Surrénalectomie: les rats sont utilisés dès la fin de la surrénalectomie ou d'une pseudoopération. Le sang est prélevé avant et une heure après l'injection de $\mathrm{I} \mathrm{mg} / \mathrm{kg}$ d'adrénaline par voie sous-cutanée. 
-- Néphrectomie: de la même façon, les rats sont utilisés dès la fin de la néphrectomie ou d'une pseudo-opération. Une seule prise de sang est effectuée I heure après l'injection d'adrénaline $(0,2 \mathrm{mg} / \mathrm{kg}$ par voie intraveineuse) ou d'un volume équivalent de sérum physiologique.

Dans le plasma hépariné, on dose le magnésium par absorption atomique (appareil PerkinElmer 303, Dawson et Heaton, I96I) et dans certains cas le glucose (glucose oxydase, MicheL, I97I) et le lactate (HoHorst, I965).

\section{RÉSULTATS}

\section{r. - Etude pharmacologique}

a) Comparaison de l'effet de l'adrénaline (effet $\alpha$ et $\beta$ ) à celui de l'isoprénaline (effet purement $\beta$ ).

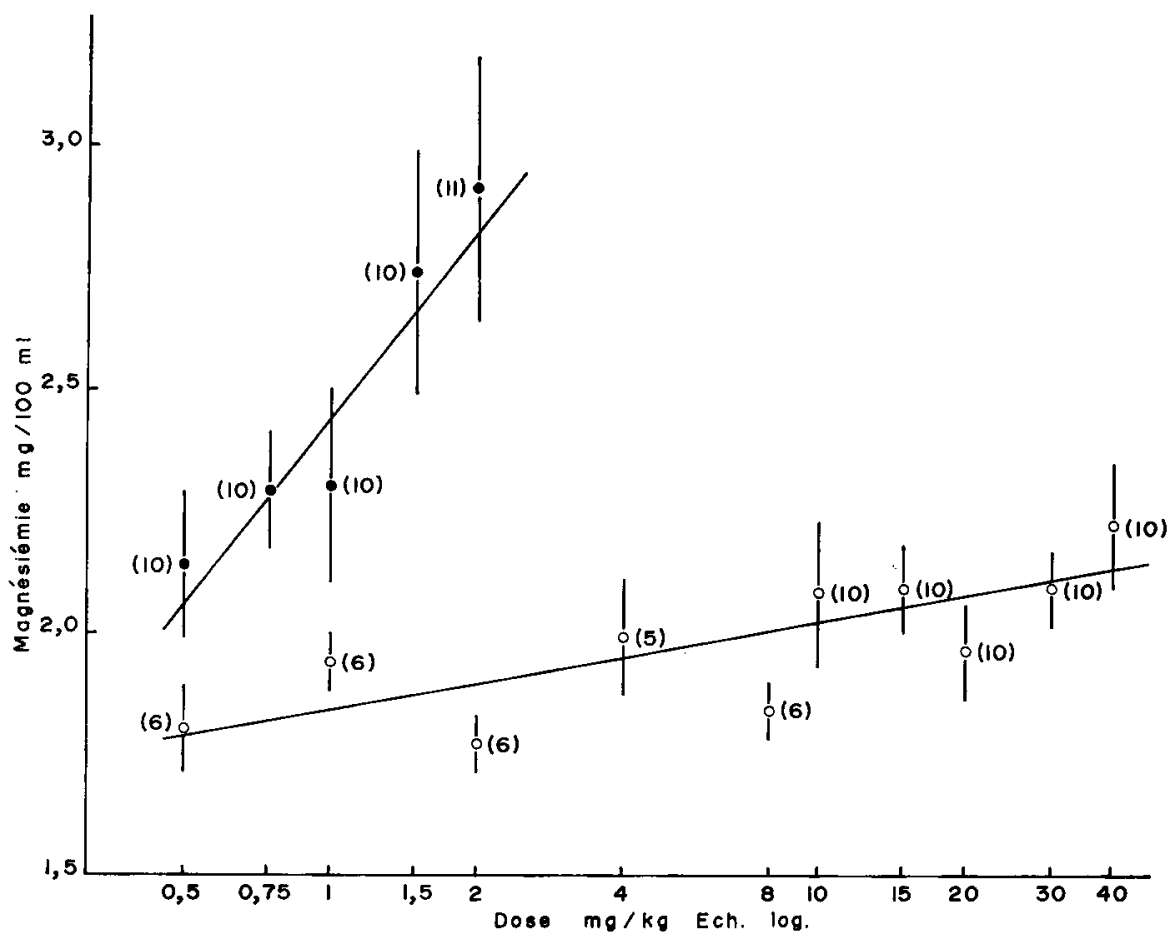

FIG. I. - Comparaison de la régression log dose-réponse pour l'effet sur la magnésémie de l'adrénaline et de l'isoprotérénol

On a figuré pour chaque lot la moyenne \pm l'erreur type, et, entre parenthèses, le nombre d'animaux. Les pentes sont significativement différentes $(P<0$,oor) - adrenaline

0 isoprénaline

FIG. I. - Comparison of the log dose-response regression for adrenaline and isoproterenol effect on magnesaemia

Mean $\pm \mathrm{S}$. E., between parentheses number of animals in each group. Slopes are significatively different $P<0,00 I)$

- adrenalin?

$\circ$ isoprenaline 
P. I,ARVOR, Y. RAYSSIGUIER

흘

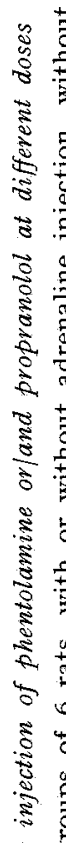

尊

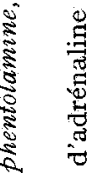

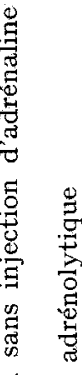

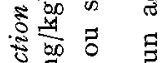

.

है

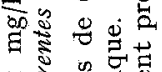

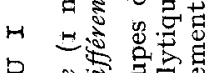

? \&

究心 $心$

तै

\&

ह

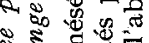

षै है

ริำ

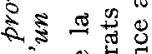

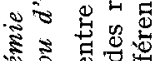

峦 ร

है

․ㅗㅀ

要

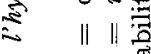

ช

\&

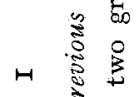

घ हो प्

崩

के

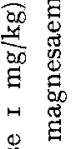

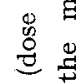

莡

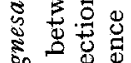

है

홍.

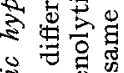

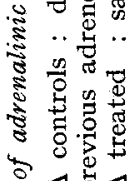

1

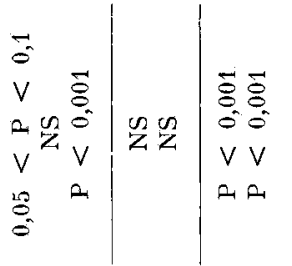

弯

至

in

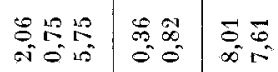

\begin{tabular}{ll|l|l|l|l|}
$\|$ & $\|$ & $\|$ & $\|$ & $\|$ & $\|$
\end{tabular}

$x^{\infty}$

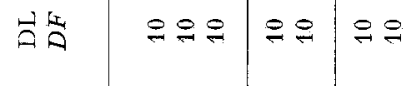

है

(3)

हิ

s

$\frac{5}{3}$

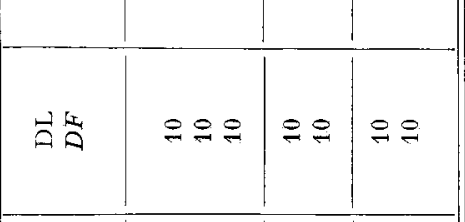

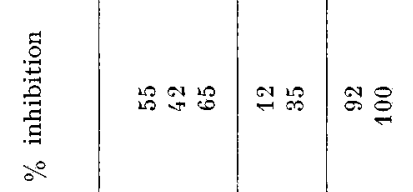

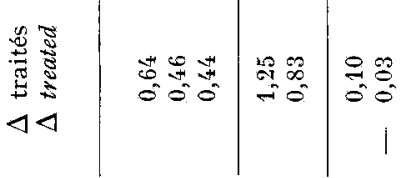

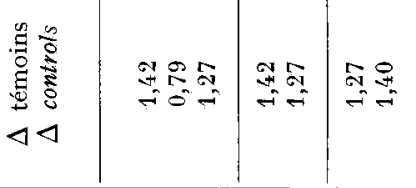

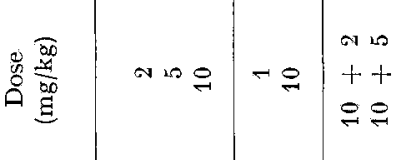

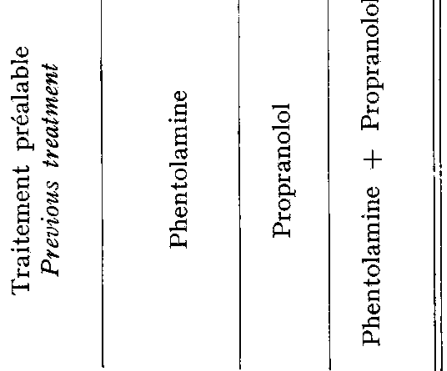




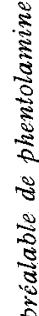

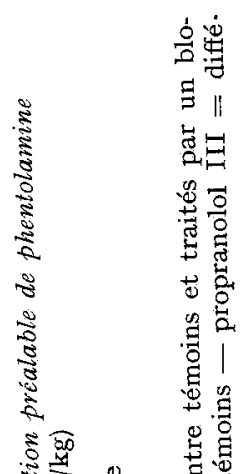

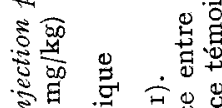

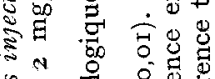

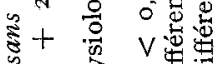

호욜

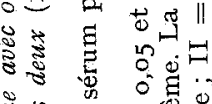

过

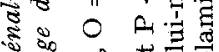

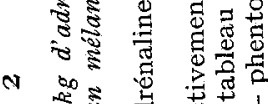

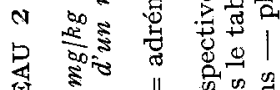

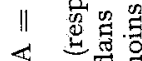

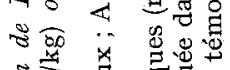

:

兽兽总

$\cdot \frac{5}{2}$

寉.

可 苛

के है

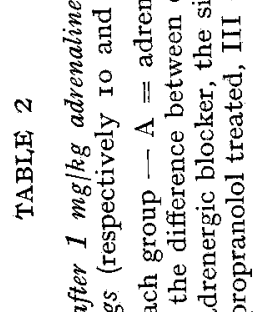

赵总离

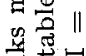

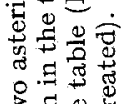

ऽ

론 귬

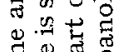

今.

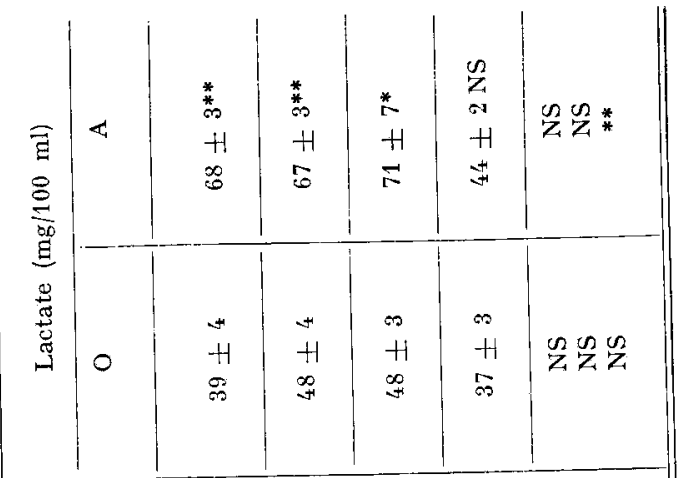

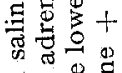

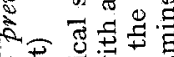

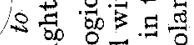

च

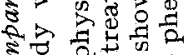

है요에

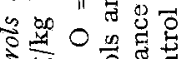

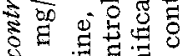

N 730

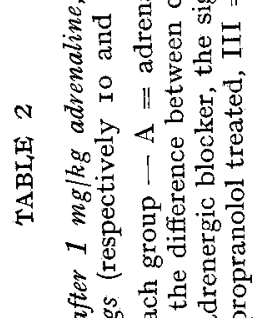

क्षे

氜

है है

늉 월

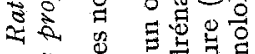

형

₹

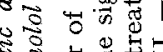

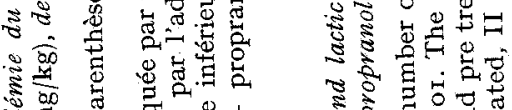

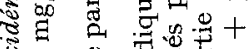

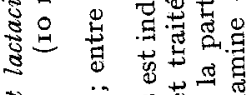

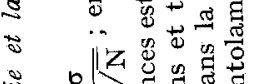

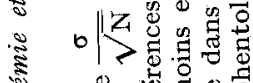

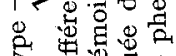

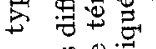

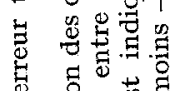

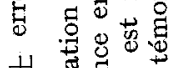

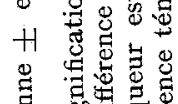

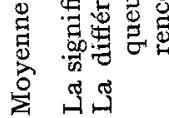

हर

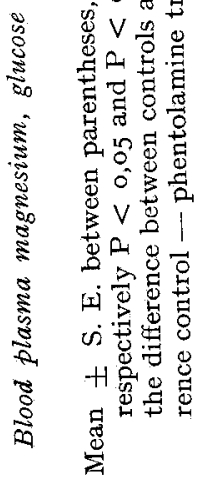

\begin{tabular}{|c|c|c|c|c|c|c|}
\hline 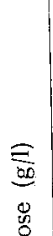 & $\varangle$ & 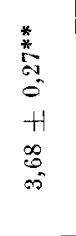 & $\begin{array}{l}0 \\
z \\
0 \\
2 \\
0 \\
H \\
0 \\
0 \\
-1 \\
-1\end{array}$ & $\begin{array}{l}* \\
\stackrel{*}{*} \\
\stackrel{+}{*} \\
0 \\
+1 \\
0 \\
0 \\
+\infty \\
\infty\end{array}$ & $\begin{array}{l}n \\
2 \\
8 \\
8 \\
0 \\
+1 \\
\infty \\
\infty \\
=-1\end{array}$ & * 䂟* \\
\hline & 0 & \begin{tabular}{l}
8 \\
8 \\
0 \\
$H$ \\
$H$ \\
$\infty$ \\
\hdashline \\
\hdashline
\end{tabular} & $\begin{array}{l}9 \\
\theta \\
+ \\
+1 \\
\infty \\
\Rightarrow\end{array}$ & $\begin{array}{l}g \\
8 \\
H \\
+ \\
\stackrel{0}{-1} \\
-1\end{array}$ & $\begin{array}{l}18 \\
0 \\
0 \\
+1 \\
0 \\
=\end{array}$ & * 贻* \\
\hline $\begin{array}{l}\overline{\bar{g}} \\
\frac{8}{8} \\
\frac{1}{00} \\
\underline{\Xi}\end{array}$ & $\varangle$ & 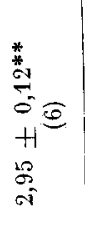 & 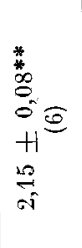 & $\begin{array}{l}n \\
z \\
5 \\
+1 \\
0 \\
+1 \\
0 \\
0 \\
0 \\
0\end{array}$ & $\begin{array}{l}n \\
z \\
0 \\
0 \\
0 \\
+1 \\
0 \\
0 \\
\infty \\
-1\end{array}$ & * 若 * \\
\hline 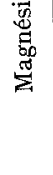 & 0 & $\begin{array}{l}8 \\
8 \\
+1 \\
+0 \\
\infty \\
\stackrel{-}{\circ}\end{array}$ & $\begin{array}{l}8 \\
0 \\
+\infty \\
5 \\
=\end{array}$ & $\begin{array}{l}8 \\
8 \\
+10 \\
+0 \\
\stackrel{0}{9} \\
\rightarrow\end{array}$ & $\begin{array}{l}8 \\
0 \\
+10 \\
0 \\
0 \\
-1\end{array}$ & 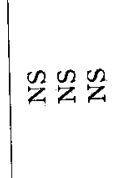 \\
\hline & & 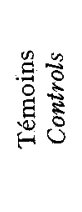 & 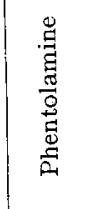 & 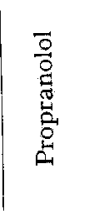 & 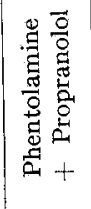 & 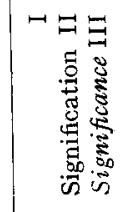 \\
\hline & & & & & & \\
\hline
\end{tabular}


L'isoprénaline utilisée en injections croissantes de $0,5 \mathrm{mg}$ à $40 \mathrm{mg}$ par $\mathrm{kg}$ de poids vif chez des rats pesant en moyenne $200 \mathrm{~g}$ entraîne une hypermagnésémie significative (fig. I). La magnésémie des témoins (Io animaux) est de I,57 $\pm 0,05 \mathrm{mg} /$ roo $\mathrm{ml}$. Chez des animaux analogues, l'adrénaline provoque également une hypermagnésémie significative. La comparaison statistique (SNEDEcor et Cochran, I967) de la pente des droites de régression de l'effet sur le logarithme de la dose pour l'adrénaline et l'isoprénaline montre une différence significative $(F=I I, 93$. $\frac{\mathrm{I}}{\mathrm{I} 36} \mathrm{DL}$ et $\mathbf{P}<0$, oor $)$.

b) Effet d'un blocage des récepteurs $\alpha$ et/ou $\beta$ sur la stimulation par l'adrénaline.

La phentolamine (bloqueur $\alpha$ ) ou le propranolol (bloqueur $\beta$ ) ont été injectés. à des doses variables avant l'administration d'adrénaline ( $\mathrm{I} \mathrm{mg} / \mathrm{kg}$ ) à des rats en lots homogènes (moyenne comprise entre $\mathrm{I} 20$ et $280 \mathrm{~g}$ selon les essais). Le tableau $\mathrm{I}$ récapitule les résultats obtenus sur la magnésémie : la phentolamine (Io $\mathrm{mg} / \mathrm{kg}$ ) inhibe significativement l'hypermagnésémie due à l'adrénaline $(\mathrm{P}<0,00 \mathrm{I})$. Le propranolol ( I et Io $\mathrm{mg} / \mathrm{kg}$ ) est peu actif. Le mélange de phentolamine et de propranolol (Io +2 ou Io $+5 \mathrm{mg} / \mathrm{kg}$ ) inhibe presque totalement 1'hypermagnésémie. Le tableau 2 montre l'effet de l'association de ces 2 bloqueurs adrénergiques sur la magnésémie, la glycémie et la lactacidémie. Les résultats sur la magnésémie sont conformes aux données des essais précédents. La phentolamine seule (Io $\mathrm{mg} / \mathrm{kg}$ ) empêche 1'hyperglycémie adrénalinique mais n'abaisse pas la lactacidémie. Le propranolol seul (2 $\mathrm{mg} / \mathrm{kg}$ ) n'inhibe pas l'hyperglycémie ni l'hyperlactacidémie. L'association des deux bloque aussi bien les effets sur le glucose et sur le lactate que sur la magnésémie.

c) Infuence d'un blocage $\alpha$ etlou $\beta$ sur les effets de l'isoprénaline.

L'injection de Io $\mathrm{mg} / \mathrm{kg}$ de propranolol inhibe significativement $(\mathbf{P}<0$,oor) l'hypermagnésémie et 1'hyperlactacidémie après isoprénaline à la dose de $20 \mathrm{mg} / \mathrm{kg}$

TABLEAU 3

Magnésémie, glycémie et lactacidémie du Rat après injection d'isoprénaline (2o $\mathrm{mg} / \mathrm{kg}$ ) avec ou sans injection préalable de $10 \mathrm{mg} / \mathrm{hg}$ de propranolol

(Même expression des résultats que pour le tableau 2)

TABI,E 3

Magnesaemia, glycaemia and lactacidaemia in rat after isoprenaline injection (2o $\mathrm{mg} / \mathrm{kg}$ ) with or without previous injection of propranolol (ro $\mathrm{mg} / \mathrm{kg}$ )

(Same expression of results than for table 2)

\begin{tabular}{|c|c|c|c|c|c|c|}
\hline \multirow[b]{2}{*}{$\begin{array}{l}\text { Témoins } \\
\text { Controls }\end{array}$} & \multicolumn{3}{|c|}{$\mathrm{O}$} & \multicolumn{3}{|c|}{ Isoprénaline } \\
\hline & $\begin{array}{c}\text { Magnésium } \\
1,54 \frac{ \pm}{(7)} 0,04\end{array}$ & $\begin{array}{c}\text { Glucose } \\
1,24 \pm 0,12\end{array}$ & $\mid \begin{array}{l}\text { Lactate } \\
33 \pm 3\end{array}$ & $\begin{array}{c}\text { Magnésium } \\
2,29 \pm \underset{(7)}{ \pm} 0,07^{* *}\end{array}$ & $\begin{array}{c}\text { Glucose } \\
1,23 \pm 0,07 \mathrm{NS}\end{array}$ & $\begin{array}{c}\text { Lactate } \\
56 \text { 土 } 3^{* *}\end{array}$ \\
\hline Propranolol & $1,59 \underset{(7)}{ \pm} 0,05$ & $1,65 \pm 0,07$ & $24 \pm 4$ & $1,87 \pm \frac{17)}{(7)} 0,4^{* *}$ & $1,31 \pm 0,16 \mathrm{NS}$ & $3 t \pm 3 \mathrm{NS}$ \\
\hline $\begin{array}{l}\text { Signification } \\
\text { Significance }\end{array}$ & NS & $*$ & NS & $* *$ & NS & $* *$ \\
\hline
\end{tabular}


chez des rats d'un poids moyen de $\mathrm{I} 60 \mathrm{~g}$ (tab1. 3). Le propranolol a un léger effet hyperglycémiant. L'isoprénaline n'élève pas la glycémie.

L'injection de ro $\mathrm{mg} / \mathrm{kg}$ de phentolamine, qui réduit l'hypermagnésémie adrénalinique, n'empêche pas l'élévation de la magnésémie après isoprénaline comme le montre le tableau 4 (rats d'un poids moyen de I7o g). Le mélange phentolamine (Io $\mathrm{mg} / \mathrm{kg}$ ) et propranolol $(5 \mathrm{mg} / \mathrm{kg}$ ) inhibe presque totalement 1'hypermagnésémie par l'isoprénaline.

\section{TABLEAU 4}

Magnésémie du Rat après injection de $20 \mathrm{mg} / \mathrm{kg}$ d'isoprénaline avec ou sans injection préalable de phentolamine (10 $\mathrm{mg} / \mathrm{kg}$ ) ou d'un mélange de phentolamine (10 $\mathrm{mg} / \mathrm{kg}$ ) et de propranolol $(5 \mathrm{mg} / \mathrm{kg}$ )

(Même expression des résultats que pour le tableau 2)

TABLE 4

Magnesaemia of the rat after isoprenaline injection $(20 \mathrm{mg} / \mathrm{kg}$ ) with or without previous injection of phentolamine (1o $\mathrm{mg} / \mathrm{kg}$ ) or phentolamine + propranolol (10 $+5 \mathrm{mg} / \mathrm{kg}$ )

(Same expression of results than for table 2)

\begin{tabular}{|c|c|c|}
\hline & $\mathrm{O}$ & Isoprénaline \\
\hline $\begin{array}{l}\text { Témoins } \\
\text { Controls }\end{array}$ & $1,73 \underset{(6)}{ \pm} 0,07$ & $2,25 \pm \underset{(6)}{0,09 * *}$ \\
\hline Phentolamine & $1,72 \underset{(6)}{ \pm} 0,0^{\prime}$ & $2,26 \underset{(6)}{ \pm} 0,19^{*}$ \\
\hline $\begin{array}{l}\text { Phentolamine } \\
+ \text { Propanolol }\end{array}$ & $1,82 \underset{(6)}{ \pm} 0,11$ & $1,93 \pm \underset{(6)}{0,05 \mathrm{NS}}$ \\
\hline Signification I & NS & NS \\
\hline Significance II & NS & * \\
\hline
\end{tabular}

\section{TABLEAU 5}

Effet de différentes doses d'isoprénaline

sur la magnésémie du Rat de poids inférieur à $100 \mathrm{~g}$

(Même expression des résultats que pour le tableau 2. Les différences ne sont pas significatives.)

TABLE 5

Effect of various isoprenaline doses

on rat magnesaemia with rat weighing less than $100 \mathrm{~g}$

(Same expression of results than for table 2. Differences are not significant)

\begin{tabular}{|c|c|c|c|c|c|c|}
\hline Dose $\mathrm{mg} / \mathrm{kg}$ & 0 & 10 & 15 & 20 & 30 & 40 \\
\hline $\mathbf{M g}$ plasma & $1,64 \underset{(10)}{ \pm} 0,06$ & $1,69 \underset{(10)}{ \pm} 0,05$ & $1,64 \underset{(10)}{ \pm} 0,04$ & $1,75 \underset{(10)}{ \pm} 0,06$ & $1,80 \underset{(10)}{ \pm} 0,08$ & $1,61 \underset{(10)}{ \pm} 0,04$ \\
\hline
\end{tabular}


d) Cas particulier des jeunes rats.

Chez des jeunes rats de poids inférieur à $\mathrm{Ioo} g$, des résultats différents ont été obtenus. L'injection d'isoprénaline, même à forte dose $(40 \mathrm{mg} / \mathrm{kg})$, n'élève pas la magnésémie de ces animaux (tabl. 5) contrairement à l'administration d'adrénaline ( $\mathrm{mg} / \mathrm{kg}$ ) (tabl. 6). Mais en comparant les tableaux I et 6 on peut remarquer que l'accroissement de la magnésémie après une injection de $\mathrm{Img} / \mathrm{kg}$ d'adrénaline $(\Delta \mathrm{Mg})$ est significativement plus faible $(\Delta \mathrm{Mg}=0,47)$ que chez des animaux plus lourds $(\Delta \mathrm{Mg}=\mathrm{I}, 22)(\mathrm{P}<0,05)$. Le traitement préalable par un adrénolytique, soit phentolamine (ro $\mathrm{mg} / \mathrm{kg}$ ) soit propranolol $(5 \mathrm{mg} / \mathrm{kg}$ ), ne permet pas de réduire l'hypermagnésémie de ces jeunes rats.

\section{TABLEAU 6}

Essai d'inhibition de l'hypermagnésémie après adrénaline ( $\mathrm{I} \mathrm{mg} / \mathrm{kg}$ ) par injection préalable de phentolamine ou de propranolol sur des rats de poids inférieur à $100 \mathrm{~g}$

$\Delta$ témoins : différence entre la magnésémie de 2 groupes de 6 rats avec ou sans injection d'adrénaline

$\Delta$ traités : idem chez des rats traités par un adrénolytique.

TABLE 6

Attempts to inhibit adrenaline hypermagnesaemia ( $\mathrm{I} \mathrm{mg} / \mathrm{kg}$ ) by previous injection of phentolamine or propranolol to rats weighing less than $100 \mathrm{~g}$

$\Delta$ controls : difference between the magnesaemia of 2 groups of 6 rats each, with or without adrenaline

$\Delta$ treated : idem with previous adrenolytic treatment.

\begin{tabular}{c|c|c|c|c}
\hline $\begin{array}{c}\text { Traitement } \\
\text { Treatment }\end{array}$ & $\begin{array}{c}\text { Dose } \\
(\mathrm{mg} / \mathrm{kg})\end{array}$ & $\begin{array}{c}\Delta \text { témoins } \\
\Delta \text { controls }\end{array}$ & $\begin{array}{c}\Delta \text { traités } \\
\Delta \text { treated }\end{array}$ & $\begin{array}{c}\text { Signification } \\
\text { Significance }\end{array}$ \\
\hline Phentolamine & 5 & 0,31 & 0,26 & $\mathrm{NS}$ \\
\hline Propranolol & 10 & 0,63 & 1,04 & $\mathrm{NS}$ \\
\hline
\end{tabular}

\section{2. - Localisation anatomique des récepteurs}

a) Éviscération abdominale (sauf le foie, le rein et les surrénales).

La magnésémie, la glycémie et la lactacidémie sont assez élevées après éviscération partielle. Chez des rats d'un poids moyen de $350 \mathrm{~g}$, l'injection sous-cutanée de $2 \mathrm{mg} / \mathrm{kg}$ d'adrénaline entraîne une hypermagnésémie et une hyperlactacidémie significatives $(\mathrm{P}<0,00 I)$ et le maintien de la glycémie à une valeur élevée alors que celle-ci tend à diminuer chez des animaux témoins (tabl. 7).

b) Hépatectomie partielle.

Chez des rats de $200 \mathrm{~g}$ ayant subi une hépatectomie partielle de $60 \mathrm{p}$. Ioo de la masse hépatique, 24 heures auparavant, une injection sous-cutanée de $2 \mathrm{mg} / \mathrm{kg}$ d'adrénaline provoque une augmentation de la magnésémie qui n'est pas significativement différente de celle observée chez des animaux pseudo-opérés. L'élévation la glycémie est, par contre, très différente dans les deux groupes d'animaux $(\mathrm{P}<0, \mathrm{OoI})($ tab1. 8). 


\section{TABLEAU 7}

Influence de l'éviscération abdominale (sauf le foie, les reins et les surrénales), sur la magnésémie, la glycémie et la lactacidémie du Rat avant et $60 \mathrm{mn}$ après l'injection d'adrénaline $(2 \mathrm{mg} / \mathrm{kg}$ sous-cutanée)

(Même expression des résultats que pour le tableau 2)

\section{TABLE 7}

Influence of abdominal evisceration (except liver kidneys and adrenals) on magnesaemia, glycaemia and lactacidaemia of rat, before and $60 \mathrm{mn}$ after adrenaline injection ( $2 \mathrm{mg} / \mathrm{kg}$ subcutaneous)

(Same expression of results than for table 2)

\begin{tabular}{|c|c|c|c|c|c|c|}
\hline \multirow[b]{2}{*}{$\begin{array}{l}\text { Temps } \\
\text { Time }\end{array}$} & \multicolumn{2}{|c|}{ Magnésium } & \multicolumn{2}{|c|}{ Glucose } & \multicolumn{2}{|c|}{ Lactate } \\
\hline & 0 & $60 \mathrm{mn}$ & 0 & $60 \mathrm{mn}$ & 0 & $60 \mathrm{mn}$ \\
\hline $\begin{array}{l}\text { Témoins } \\
\text { Controls } \\
\text { Adrénaline }\end{array}$ & $\mid \begin{array}{c}2,13 \underset{(6)}{ \pm} 0,07 \\
2,03 \underset{(6)}{ \pm} 0,04\end{array}$ & $\begin{array}{c}2,55 \pm 0,27 \mathrm{NS} \\
(6) \\
3,71 \pm 0,33^{* *} \\
(6)\end{array}$ & $\begin{array}{l}2,55 \pm 0,45 \\
2,19 \pm 0,32\end{array}$ & $\begin{array}{l}1,75 \pm 0,43 \mathrm{NS} \\
2,01 \pm 0,63 \mathrm{NS}\end{array}$ & $\begin{array}{l}52 \pm 4 \\
54 \pm 4\end{array}$ & $\begin{array}{l}50 \pm 6 \mathrm{NS} \\
75 \pm 2^{* *}\end{array}$ \\
\hline $\begin{array}{l}\text { Signification } \\
\text { Significance }\end{array}$ & NS & $* *$ & NS & NS & NS & $* *$ \\
\hline
\end{tabular}

\section{TABLEAU 8}

Influence de l'hépatectomie partielle sur l'accroissement de la magnésémie ( $\Delta \mathrm{Mg}$ ) et de la glycémie ( $\Delta$ glucose) après injection d'adrénaline $(2 \mathrm{mg} / \mathrm{kg}$ sous-cutanée $)$ et influence do la surrénalectomie sur l'effet de l'adrénaline (1 $\mathrm{mg} / \mathrm{kg}$ sous-cutanée)

\section{TABLE 8}

Effect of adrenaline (2 $\mathrm{mg} / \mathrm{kg}$ subcutaneous) on magnesaemia and glycaemia after partial hepatectomy,

and of adrenaline ( $\mathrm{m} \mathrm{mg} / \mathrm{kg}$ subcutaneous) on magnesaemia after adrenalectomy

\begin{tabular}{c|c|c|c|c|c|c}
\hline \hline & \multicolumn{5}{|c|}{$\begin{array}{c}\text { Hépatectomie } \\
\text { Hepatectomy }\end{array}$} & \multicolumn{2}{c}{$\begin{array}{c}\text { Surrénalectomie } \\
\text { Adrenalectomy }\end{array}$} \\
\cline { 2 - 6 } & Nombre & $\%$ ablation & $\Delta \mathrm{Mg}$ & $\Delta$ glucose & Nombre & $\Delta \mathrm{Mg}$ \\
\hline $\begin{array}{c}\text { Pseudo-opérés } \\
\text { Sham operated }\end{array}$ & 7 & 0 & $0,79 \pm 0,14$ & $1,84 \pm 0,21$ & 7 & $0,69 \pm 0,12$ \\
\hline $\begin{array}{c}\text { Opérés } \\
\text { Operated }\end{array}$ & 5 & $59,8 \pm 3,0$ & $1,03 \pm 0,20$ & $0,36 \pm 0,19$ & 6 & $0,65 \pm 0,16$ \\
\hline $\begin{array}{c}\text { Signification } \\
\text { Significance }\end{array}$ & & & NS & $* *$ & & $\mathrm{NS}$ \\
\hline \hline
\end{tabular}


c) Surrénalectomie.

L'hypermagnésémie chez des rats de $200 \mathrm{~g}$ ayant reçu une injection sous-cutanée de I mg/kg d'adrénaline, n'est pas influencée par la surrénalectomie réalisée juste avant 1'injection (tabl. 8).

d) Néphrectomie.

L'hypermagnésémie obtenue par 1'injection intraveineuse de $0,2 \mathrm{mg} / \mathrm{kg}$ d'adrénaline chez des rats pesant en moyenne $35^{\circ} \mathrm{g}$ n'est pas significativement différente chez des animaux néphrectomisés ou pseudo-opérés (tab1. 9).

\section{TABLEAU 9}

Effet de la néphrectomie sur la magnésémie du Rat après injection d'adrénalina $(0,2 \mathrm{mg} / \mathrm{kg} \mathrm{IV})$

(Même expression des résultats que pour le tableau 2) TABLE 9

Effect of nephrectomy on adrenalinic hypermagnesaemia $(0,2 \mathrm{mg} / \mathrm{kg}$ intravenous)

(Same expression of results than for table 2)

\begin{tabular}{c|c|c}
\hline & 0 & Adrénaline \\
\hline $\begin{array}{c}\text { Pseudo-opérés } \\
\text { Sham operated }\end{array}$ & $1,43 \frac{ \pm}{(5)} 0,06$ & $2,34 \pm 0,29^{* *}$ \\
\hline $\begin{array}{c}\text { Opérés } \\
\text { Operated }\end{array}$ & $1,48 \frac{ \pm}{(5)} 0,09$ & $2,20 \pm 0,38 \mathrm{NS}$ \\
\hline $\begin{array}{c}\text { Signification } \\
\text { Significance }\end{array}$ & $\mathrm{NS}$ & $\mathrm{NS}$ \\
\hline \hline
\end{tabular}

\section{DISCUSSION}

L'existence d'une différence significative dans la pente de la régression log-doseréponse pour l'adrénaline et l'isoprénaline signifie que le mode d'action des deux substances n'est pas de la même nature en ce qui concerne l'élévation de la magnésémie. Des essais d'inhibition de l'hypermagnésémie par des adrénolytiques spécifiques montrent également des différences importantes entre 1'isoprénaline et l'adrénaline. Ainsi le propranolol, bloqueur $\beta$ peu actif avec l'adrénaline, abaisse la magnésémie après isoprénaline tandis que la phentolamine, bloqueur $\alpha$ qui réduit 1'hypermagnésémie adrénalinique, n'empêche pas l'élévation de la magnésémie après isoprénaline. La nécessité d'une combinaison d'adrénolytiques $\alpha$ et $\beta$ pour bloquer certains effets des catécholamines est désormais classique (AHLQUIST et LÉvY, I959). Dans tous les cas, après adrénaline ou isoprénaline, une inhibition presque totale est obtenue par un mélange de phentolamine et de propranolol alors que selon les cas la phentolamine ou le propranolol n'entraînent qu'une inhibition partielle. 
Après ces différents essais, il reste difficile de caractériser les récepteurs adrénergiques de l'hypermagnésémie. Les pouvoirs respectifs de l'adrénaline, de la noradrénaline et de l'isoprénaline, selon la terminologie d'AHL,QUIST (I948) font penser à un effet $\beta$. L'action du propranolol après isoprénaline renforce cette hypothèse mais il apparaît que les actions de l'adrénaline et de l'isoprénaline sont de type différent (au moins en partie) et, en ce qui concerne l'effet de l'adrénaline, le blocage décevant de l'hypermagnésémie par le propranolol, l'action de la phentolamine et l'inhibition totale par une combinaison de bloqueurs $\alpha$ et $\beta$ ne clarifient pas les choses. L'existence de récepteurs adrénergiques d'un type particulier étant désormais admise chez le Rat pour un certain nombre d'effets métaboliques des catécholamines (élévation de la glycémie, des acides gras libres), l'ensemble de nos résultats semblerait montrer la présence de récepteurs $\beta$ non typiques. En réalité, on peut se poser le problème de savoir si la distinction entre récepteurs $\alpha$ et $\beta$ garde un sens dans ce cas précis.

I1 est intéressant de noter les rapports des récepteurs adrénergiques responsables de 1'hypermagnésémie avec ceux qui entraînent l'hyperglycémie et 1'hyperlactacidémie classiquement observées après une injection de catécholamines. On sait que l'hyperglycémie est essentiellement due à la glycogénolyse hépatique car la glucose-6-phosphatase n'existe que dans le tissu hépatique ou rénal tandis que l'hyperlactacidémie est due à la glycogénolyse musculaire.

Nos résultats confirment ceux de Eliris (1967) selon lequel les récepteurs de la glycogénolyse hépatique ne sont pas des récepteurs $\beta$ typiques chez le Rat. Ainsi 1'isoprénaline n'augmente pas la glycémie dans nos essais en accord avec KENNY (I965). Au contraire l'isoprénaline entraîne une hypermagnésémie significative. Le propranolol ne prévient pas l'hyperglycémie et augmente légèrement la glycémie d'animaux non traités par des catécholamines. BLINKS a montré (I967) que même le propranolol peut avoir un effet sympathomimétique, il est possible qu'avec la dose élevée (Io $\mathrm{mg} / \mathrm{kg}$ ) employée nous soyons dans ce cas. D'une façon générale les bloqueurs adrénergiques $\beta$ sont considérés comme de mauvais inhibiteurs de 1'hyperglycémie près adrénaline chez le Rat (CLAASSEN et NoACH, I96o ; HoRNBRook et BRODY, I963). La phentolamine inhibe dans tous les cas et totalement l'hyperglycémie alors que son action sur la magnésémie est inconstante et partielle. Ces résultats montrent les différences qui existent entre les récepteurs responsables de la glycogénolyse hépatique et les récepteurs de l'hypermagnésémie. La glycogénolyse musculaire est contrôlée chez le Rat par des récepteurs $\beta$ adrénergiques typiques. L'hyperlactacidémie apparaît après injection d'adrénaline et d'isoprénaline. L'inhibition peut être obtenue par des bloqueurs $\beta$ et non par des bloqueurs des $\alpha$ récepteurs en accord avec HoRNBROOK et BRODY (I963), KENNEDY et EILIIS (I963). Il semble donc que l'on puisse aussi dissocier, au moins en partie, les récepteurs responsables de l'hypermagnésémie et les récepteurs de la glycogénolyse musculaire.

En ce qui concerne les essais de localisation anatomique des récepteurs adrénergiques, l'hépatectomie confirme les résultats pharmacologiques qui permettent de différencier les récepteurs responsables de l'hypermagnésémie et de l'hyperglycémie. L'ablation d'au moins 60 p. Ioo du foie, n'influe pas sur l'hypermagnésémie adrénalinique alors que l'hyperglycémie, conséquence d'une glycogénolyse exclusivement hépatique, est très largement inhibée. Il est permis également d'exclure un mécanisme pour l'hypermagnésémie voisin de celui décrit par D'Sirva (I934) pour l'hyperka- 
liémie après injection de catécholamines puisque dans ce dernier cas le foie est à l'origine de la libération du potassium. Après éviscération abdominale (sans ablation du foie, des reins et des surrénales), la magnésémie, la glycémie et la lactacidémie sont assez élevées. Le traumatisme qui découle de toute intervention chirurgicale dans l'abdomen est ici particulièrement important avec une forte stimulation sympathique et glycogénolyse musculaire et hépatique. La glycémie tend à s'abaisser dans l'heure qui suit tandis que la magnésémie s'élève légèrement. L'injection d'adrénaline a peu d'effet sur la glycémie car les réserves en glycogène hépatique ont déjà été sollicitées mais élève fortement la magnésémie.

L'ablation des reins et des surrénales n'empêche pas davantage 1'hypermagnésémie après adrénaline.

Ces résultats montrent que les récepteurs adrénergiques responsables de l'hypermagnésémie ne doivent pas être recherchés dans les viscères abdominaux puisque l'élévation du magnésium plasmatique se produit en leur absence et que ces organes ne sont pas la source de l'augmentation de la magnésémie ou du moins n'en constituent pas l'origine principale.

En conclusion, l'hypermagnésémie consécutive à l'injection d'adrénaline ou d'isoprénaline chez le Rat présente des caractéristiques physiologiques assez complexes : les mécanismes d'action de ces deux substances ne sont pas entièrement superposables, la pente de la relation dose-réponse est significativement différente et les caractéristiques du blocage pharmacologique sont différentes.

L'isoprénaline paraît agir sur des récepteurs $\beta$ typiques, tandis que l'adrénaline, dont 1'action est nettement plus puissante, semble agir à la fois sur des récepteurs analogues à ceux de l'isoprénaline et sur des récepteurs $\beta$ d'un type particulier, qui ne peuvent être bloqués efficacement que par la conjonction de bloqueurs $\alpha$ et $\beta$ (phentolamine + propranolol). Les récepteurs sensibles à l'isoprénaline n'existent pas chez des jeunes rats de moins de roo $\mathrm{g}$ qui sont également moins sensibles à l'adrénaline ; cependant cette hypermagnésémie plus faible est aussi plus difficile à bloquer par les adrénolytiques.

L'hypermagnésémie adrénalinique, effet métabolique typique, est mettement dissociable de la glycogénolyse hépatique au moyen de blocages pharmacologiques sélectifs ou de l'hépatectomie partielle. Ses caractéristiques pharmacologiques paraissent également la dissocier, au moins en partie, de la glycogénolyse musculaire.

On peut éliminer les viscères abdominaux comme source possible de magnésium ou relais obligatoires pour la mise en circulation de cet élément. Par voie de conséquence, l'hypermagnésémie adrénalinique ne résulte pas essentiellement d'une résorption intestinale ou d'une rétention rénale accrues.

Reçu pour publication en novembre 1970.

\section{SUMMARY}

\section{DETERMINATION OF THE RECEPTORS RESPONSIBLE FOR CATECHOLAMINE-INDUCED HYPERMAGNESAEMIA}

Intraperitoneally injected adrenaline induces considerable hypermagnesaemia in the rat, contrary to noradrenaline which has no effect on magnesaemia (LARvoR, 1968). This investigation was undertaken in order to determine the adrenergic receptors responsible for the increase in 
magnesaemia using $\alpha$-and $\beta$-stimulators and specific adrenolytic agents. Attemps were made to locate the receptors sites using surgical excisions.

The blood was collected by cardiac puncture. In the heparine-treated plasma, the magnesium rate was estimated by atomic absorption, glucose by glucose oxydase and lactate by an enzymatic technique.

\section{Pharmacological study.}

Blocking phentolamine $(\alpha)$ or propranolol $(\beta)$ were intraperitoneally injected $30 \mathrm{mn}$ before catecholamine injection. The blood was collected $60 \mathrm{mn}$ after administration of L-adrenaline $(\alpha$-and $\beta$-stimulators) or isoprenaline ( $\beta$-stimulator).

Graph I shows the effect of increasing adrenaline or isoprenaline doses on magnesaemia. Adrenaline is more potent than isoprenaline and a statistical comparison between the slopes of $\log /$ dose response regression curves for adrenaline and isoprenaline was significant.

Tables $I$ and 2 point out the effect of $\alpha$ - and $\beta$-receptors blockade on adrenaline stimulation. Significant inhibition of hypermagnesaemia and hyperglycemia was induced by phentolamine, little inhibition by propranolol, nearly total inhibition of hypermagnesaemia, hyperglycemia and hyperlactacidaemia by a combination of phentolamine and propranolol.

Table 3 indicates the effect of $\alpha$ - and $\beta$ receptors blockade on isoprenaline stimulation. Isoprenaline induces hypermagnesaemia but no increase in glycemia. Propranolol significantly inhibits hypermagnesaemia and hyperlactacidaemia. Phentolamine induces a decrease in adrenalineinduced but not in isoprenaline-induced hypermagnesaemia.

These results were obtain with rats of $120-280 \mathrm{~g}$ body weight. With younger rats weigning less than $100 \mathrm{~g}$, isoprenaline injection even at high rates has no effect on magnesaemia ; adrenaline affects magnesaemia to a lesser extent and blockade by adrenolytic agents (tables 5 and 6 ) is more difficult.

In view of these experiments, determination of the adrenergic receptors of hypermagnesaemia remains difficult. The specific effects of adrenaline, noradrenaline and isoprenaline were observed to be partly of different types. With rats weighing more than Ioo g, isoprenaline would seem to act on typical $\beta$-receptors inhibited by propranolol, whereas adrenaline which is more effective, could possibly act both on similar and specific receptors which could be inhibited only by a combination of $\alpha$ and $\beta$ blocking agents. Evidence for such specific receptors has been found in the rat for certain metabolic effects such as hyperglycemia.

Our results point out the differences existing between the receptors responsible for hyperglycemia (liver glycogenolysis) and those responsible for hypermagnesaemia; e.g. contrary to magnesaemia, glycemia was not increased by isoprenaline and adrenaline-induced hyperglycemia was totally inhibited by phentolamine. It also appears that hypermagnesaemic and muscular glycogenolytic receptors can be differenciated, since it was not possible to phentolamine inhibit hyperlactacidaemia, a typical $\beta$ adrenergic phenomenon.

\section{Anatomical location of the receptors.}

Rats of 250-300 g body weights were either eviscerated with the exception of liver, kidneys and adrenals, or adrenalectomized, or nephrectomized or partly hepatectomized. Our results (see tables $7,8,9)$ show that no adrenergic receptor responsible for hypermagnesaemia can be located in these organs, as the increase in plasmic magnesium rate, estimated $\mathrm{I} h$ after adrenaline injection, occurs irrespective of their presence. Therefore, hypermagnesaemia cannot be chiefly due to increased intestinal resorption or altered urinary excretion.

\section{RÉFÉRENCES BIBLIOGRAPHIQUES}

Ahlouist R. P., 1948. A study of adrenotropic receptors. Amer. J. Physiol., 153, 586-60o.

Ahlquist R. P., Levy B., r959. Adrenergic receptive mechanism of canine ileum. J. Pharmacol. Exptl. Biol. Med., 127, 146-149.

Blinks J. R., 1967. Evaluation of the cardiac effects of several beta-adrenergic blocking agents. Ann. N. Y. Acad. Sci., 139, 673-685.

Claassen V., Noach E. L., 1960. Dichloro-isuprel inhibition of sympathomimetic hyperglycaemia. Arch. int. Pharmadodyn, 126, 322-340.

Dawson J. B., Heaton E. W., r96r. The determination of magnesium in biological materials by atomic absorption spectrophotometry. Biochem. J., 80, 99-106.

D'Silva J. L., I933. The influence of adrenaline on the blood potassium. $J$. Physiol., 80, 7 p. 
Ellis S., Kennedy B. L., Eusebi A. J., Vincent N. H., 1967. Autonomic control of metabolism. Ann. N. Y. Acad. Sci., 139, 826-832.

Hohorst H. J., I965. L $(+)$ lactate determination with lactic dehydrogenase and DPN, in BERGMEYER H. V. Methods of enzymatic analysis, I vol. 266-27o, Academic Press, ed. New York.

Hornbrook K. R., Brody T. M., I963. Phosphorylase activity in rat liver and skeletal muscle after catecholamines. Biochem. Pharmacol., 12, I407-I4I5.

Ingle D. J., Griffith J. Q., 1949. Surgery of the rat in : Farris E. J., Griffith J. Q. The rat in laboratory investigation 434-45I, I vol. $2^{\mathrm{e}}$ édition, Hafner Publ. Co. New York.

Kabal J., Ramey E. R., r965. Effect of liver damage on plasma FFA response to epinephrine. Proc. Soc. Exptl. Biol. Med., 119, 708-710.

KENNEDY B. L., Ellis S., 1963. Interaction of sympathomimetic amines and adrenergic blocking agents at receptors sites mediating glycogenolysis. Fed. Proc., 22, 449.

Kenny A. D., Fleming W. W., 1965. Effect of adrenalectomy on hyperglycemic response of fed and fasted rats to catecholamines. Proc. Soc. Exptl. Biol. Med., 119, 255-258.

Larvor P., I968. Effet de l'adrénaline et de la noradrénaline sur la magnésiémie et la glycémie du rat. Interférence de la dihydroergotamine et de l'insuline. Ann. Biol. anim. Bioch. Biophys., 8, 46I-464.

Michel M.-C., 1971. Dosage du glucose et du phosphore minéral dans le sang à l'autoanalyseur Technicon. Ann. Biol. anim. Bioch. Biophys. (sous presse).

Robison G. A., Butcher R. W., Sutherland E. W., r967. Adenyl cyclase as an adrenergic receptor. Ann. N. Y. Acad. Sci., 138, 703-723.

SNedecor G. W., Cochran W. G., I967. Statistical methods. I vol., 953 p., 6 e édition. Iowa State University Press, Ames, Iowa, U. S. A. 